\title{
TRATAMENTO CONSERVADOR OCULAR DOS MELANOMAS DE CORÓIDE COM BRAQUITERAPIA UTILIZANDO PLACAS DE IODO-125*
}

\author{
Antônio Cássio Assis Pellizzon ${ }^{1,2}$, Maria Aparecida Conte Maia ${ }^{1}$, Petrus Paulo C.E. da Silva ${ }^{1}$, \\ Paulo Eduardo Ribeiro dos Santos Novaes ${ }^{1}$, Ricardo César Fogaroli ${ }^{1,2}$, João Victor Salvajoli ${ }^{1}$, \\ Robson Ferrigno ${ }^{1}$, Ricardo José Assis Pellizzon ${ }^{3}$, Marta Chodjaniack ${ }^{4}$, Márcia Motono ${ }^{4}$
}

Resumo OBJETIVO: Avaliar, retrospectivamente, o resultado do tratamento conservador do melanoma de coróide, por meio de braquiterapia, usando placas episclerais de iodo-125 (modelo 6711, Amershan). MATERIAIS E MÉTODOS: Foram avaliados 49 pacientes portadores de melanoma de coróide tratados no Hospital do Câncer, São Paulo, SP, de março de 2001 a janeiro de 2003. Os seguintes parâmetros foram analisados e correlacionados ao controle local e sobrevida: sexo, idade, dimensões da lesão, tempo de tratamento e doses no ápice e base das lesões. RESULTADOS: Com diâmetro máximo da base do tumor de $17 \mathrm{~mm}$ e altura máxima de $12 \mathrm{~mm}$, observamos que as doses na base do tumor variaram de 213 a $463 \mathrm{~Gy}$ (mediana de $347 \mathrm{~Gy}$ ) e no ápice, de 51 a $250 \mathrm{~Gy}$ (mediana de $91 \mathrm{~Gy}$ ). As taxas de preservação ocular, sobrevida livre de doença e conservação ocular, atuariais em dois anos, foram de 96\%, 93,5\% e 96,3\%, respectivamente. Através de análise univariada, o único fator prognóstico para controle local nesta análise foi a altura do tumor menor que $6 \mathrm{~mm}$ ( $p=0,0348$ ). CONCLUSÃO: A braquiterapia levou a uma taxa bastante satisfatória de controle local, confirmando que a altura do tumor é um dos fatores prognósticos importantes desse parâmetro.

Unitermos: Melanoma de coróide; Placa oftalmológica; Terapia conservadora; Enucleação; Braquiterapia.

Abstract Conservative treatment of choroidal melanomas using iodine brachytherapy.

OBJECTIVE: To retrospectively evaluate the results of conservative therapy using episcleral iodine-125 (model 6711, Amershan) plaque for brachytherapy of choroidal melanoma. MATERIALS AND METHODS: We evaluated 49 patients with choroidal melanoma treated at "Hospital do Câncer", São Paulo, Brazil, from March 2001 to January 2003. The following clinical parameters were analyzed and correlated with local control and survival rate: gender, age, lesion dimension, treatment duration, as well as doses in apex and base of the tumors. RESULTS: The maximum diameter and height of the base of the tumors treated were $17 \mathrm{~mm}$ and $12 \mathrm{~mm}$, respectively. Doses at those points ranged from 213 to $463 \mathrm{~Gy}$ (median: $347 \mathrm{~Gy}$ ) and 51 to $250 \mathrm{~Gy}$ (median: $91 \mathrm{~Gy}$ ), respectively. The actuarial rates of ocular preservation, disease free survival and ocular preservation in 2-years were $96 \%, 93.5 \%$ and $96.3 \%$, respectively. A tumor height $<6 \mathrm{~mm}$ was the only predictive factor for local control $(p=0.0348)$ in the univariate analysis. CONCLUSION: Brachytherapy achieved a very satisfactory rate of tumor local control, confirming that tumor height is one of the most important predictive factors for local control.

Key words: Choroidal melanoma; Plaque therapy; Conservative therapy; Enucleation; Brachytherapy.

\section{INTRODUÇÃO}

O melanoma de coróide (MC) é o tumor intra-ocular mais freqüente em adul-

* Trabalho realizado no Centro de Tratamento e Pesquisa Hospital do Câncer A.C. Camargo, São Paulo, SP.

1. Departamento de Radioterapia do Centro de Tratamento e Pesquisa Hospital do Câncer A.C. Camargo.

2. Serviço de Radioterapia do Instituto Arnaldo Vieira de Carvalho.

3. Departamento de Radiologia da Faculdade de Medicina de Catanduva, SP.

4. Departamento de Onco-oftalmologia do Centro de Tratamento e Pesquisa Hospital do Câncer A.C. Camargo.

Endereço para correspondência: Dr. Antônio Cássio Assis Pellizzon. Departamento de Radioterapia, Centro de Tratamento e Pesquisa Hospital do Câncer A.C. Camargo. Rua Professor Antônio Prudente, 211, Liberdade. São Paulo, SP, 01509-900. E-mail: pellizzon@aol.com

Recebido para publicação em 4/8/2003. Aceito, após revisão, em 3/10/2003. tos, sendo o seu tratamento causa de considerável controvérsia.

Para as lesões grandes, com mais de 10 mm de altura e/ou 20 a $25 \mathrm{~mm}$ no maior diâmetro da base, o tratamento usual é a enucleação, sendo que a radioterapia externa parece não ter nenhum impacto nos resultados, como demonstrado pela publicação do Collaborative Ocular Melanoma Study Group (COMS), em que mais de 1.000 pacientes, cujos olhos contendo MC classificados como grandes, eram sorteados para tratamento com enucleação ou enucleação precedida por radioterapia externa. Não se observou efeito negativo ou positivo nas taxas de mortalidade aos cinco e oito anos pós-tratamento ${ }^{(\mathbf{1})}$. Já para as lesões de médio e pequeno porte, outras técnicas, além da enucleação, como a fotocoagulação, a terapia por laser, a termoterapia, a irradiação com partículas, a radioterapia convencional e a braquiterapia com uso de placas episclerais são utilizadas na tentativa da preservação do olho e da sua função.

No Brasil, por causa da inexistência de equipamentos que produzam partículas pesadas, fica como técnica preferencial de tratamento irradiante a braquiterapia. Esta parece ser uma estratégia atraente para tratamento das lesões de pequeno e médio porte, em virtude da probabilidade de preservação do olho e consequiente manutenção da visão. A indicação de uma ou outra 
técnica, porém, guarda relação direta com o tamanho e com a localização da lesão ${ }^{(2)}$. Um outro estudo randomizado do COMS avaliou mais de 1.300 pacientes, portadores de lesões pequenas e médias, sorteados para receberem tratamento com braquiterapia utilizando iodo-125 ou enucleação. As taxas de mortalidade em até 12 anos não diferiram entre os dois subgrupos, e a partir da data daquela publicação a braquiterapia com iodo-125 tornou-se o procedimento mais freqüentemente indicado para os MC de pequeno e médio porte, bem como o iodo-125 o isótopo mais comumente utilizado na América do Norte ${ }^{(3)}$.

No Hospital A.C. Camargo iniciamos, em março de 2001, uma política institucional de tratamento conservador usando sementes de iodo-125, modelo 6711, Amershan, para o tratamento dos MC de tamanhos médio e pequeno. Até fevereiro de 2003 foram tratados 78 pacientes, incluindo, além dos pacientes portadores de MC, os portadores de metástases intra-oculares e retinoblastoma.

\section{MATERIAIS E MÉTODOS}

Esta é uma análise retrospectiva, realizada em uma única instituição, o Centro de Tratamento e Pesquisa Hospital do Câncer, São Paulo, Brasil. Do mês de março de 2001 a janeiro de 2003, 59 pacientes portadores de tumores intra-oculares foram consecutivamente tratados no Serviço de Braquiterapia e Departamento de Oncooftalmologia. Destes, 49 apresentavam MC de tamanho médio ou pequeno.

Os pacientes foram avaliados em relação às características clínicas de apresentação do tumor, tempo de tratamento, doses recebidas pelo ápice e base do tumor. Esses parâmetros foram correlacionados ao resultado clínico, ou seja, controle local, taxa de conservação ocular e sobrevida dos pacientes. O seguimento mediano destes pacientes foi de 31 (6-63) meses.

Como rotina diagnóstica, todos os pacientes eram submetidos a exames por biomicroscopia de lâmpada de fenda, oftalmoscopia indireta e ultra-sonografia ocular. Avaliações pulmonares, ósseas e hepáticas eram executadas em casos suspeitos. Não foi realizada biópsia por agulha em nenhum dos pacientes. Fotografias digitais foram realizadas com a finalidade de controle e documentação científica.

Depois do término do tratamento os pacientes eram avaliados a cada três meses no primeiro ano e a cada seis meses a partir de então. No seguimento eram executados exames como ultra-som e transiluminação, entre outros.

As análises de sobrevida foram realizadas pelo método de Kaplan-Meier, com as diferenças entre as curvas de sobrevidas avaliadas pelo método de log-rank. As diferenças entre as variáveis quantitativas foram realizadas através de testes do quiquadrado de Fischer. Adotou-se o nível de significância de $5 \%(\mathrm{p}=0,05)$ para todas as situações.

\section{RESULTADOS}

A idade dos pacientes variou dos 19 aos 91 anos (mediana de 57 anos). Observouse uma leve predominância do sexo feminino (31/49 pacientes) e de lesões à esquerda (30/49 casos). As características clínicas dos pacientes estão apresentadas na Tabela 1.

O diâmetro máximo da base das lesões variou de 5 a $20 \mathrm{~mm}$ (mediana de $14 \mathrm{~mm}$ ). A altura mediana das lesões foi de $6 \mathrm{~mm}$, variando de 2 a $10 \mathrm{~mm}$, medida por ultrasonografia.

A localização intra-ocular predominante foi a temporal, representada por 30 das 49 lesões $(61,2 \%)$. As lesões em situação nasal corresponderam a 12,2\% (6/49), ao passo que as lesões centrais e paracentrais representaram 10,2\% (5/49). Em 16,3\% (8/49) dos casos não foi possível determinar a localização primária da lesão pelos dados do prontuário.

Os tempos de permanência com as placas em posição variaram de 72 a 216 ho- ras (mediana de 134 horas), e o número de sementes utilizadas por tratamento variou com o tamanho da placa episcleral utilizada. O número mínimo de sementes utilizadas nos tratamentos foi de 6 e o máximo de 20 (mediana de 13 sementes).

O diâmetro máximo da base do tumor variou de 5 a $17 \mathrm{~mm}$ (mediana de $14 \mathrm{~mm}$ ). As doses na base do tumor, incluindo $1 \mathrm{~mm}$ de esclera, estiveram entre 213 e 463 Gy (mediana de 347 Gy)

A altura máxima das lesões variou de 3 a $12 \mathrm{~mm}$ (mediana de $6 \mathrm{~mm}$ ) e as doses no ápice vaiaram de 51 a 250 Gy (mediana de 91 Gy).

Para todos os pacientes, doses a $5 \mathrm{~mm}$ foram calculadas. A dose mediana recebida a $5 \mathrm{~mm}$ foi de $94 \mathrm{~Gy}$ (variação de 60 a 177 Gy).

A taxa de sobrevida livre de doença atuarial em dois anos foi de 93,5\% (Figura 1), com taxa bruta de conservação ocular de $96 \%$.

$\mathrm{Na}$ análise univariada, gênero, idade acima de 55 anos, tempo de tratamento, dose recebida pela base do tumor básico, dose a $5 \mathrm{~mm}$ e dose no ápice não foram fatores prognósticos para controle local (Tabela 2).

O único fator prognóstico de controle local nesta análise foi a altura do tumor menor que $6 \mathrm{~mm}(\mathrm{p}=0,0348)$ (Figura 2).

Três pacientes apresentaram falha no controle local, todos portadores de lesão com altura superior a $6 \mathrm{~mm}$. Dois pacientes apresentaram progressão da doença após 9 e 11 meses do término do tratamento, sendo submetidos a enucleação.

\section{DISCUSSÃO}

A enucleação como tratamento primário do MC tem sido questionada desde os

Tabela 1 Características clínicas dos pacientes.

\begin{tabular}{|l|r|r|r|r|}
\hline \multicolumn{1}{|c|}{ Variável } & № de pacientes & Porcentagem & Mediana & Variação \\
\hline Sexo Masculino & 31 & $63,3 \%$ & & \\
Feminino & 18 & $36,7 \%$ & & \\
Idade (anos) & & & 55,2 & $9-91$ \\
Diâmetro da base (mm) & & & 14,0 & $5-20$ \\
Ápice (mm) & & & 6,0 & $2-10$ \\
Dose na base (Gy) & & & 347,0 & $213-463$ \\
Dose no ápice (Gy) & & & 144,0 & $72-116$ \\
Tempo de tratamento (horas) & & & & $51-250$ \\
\hline
\end{tabular}




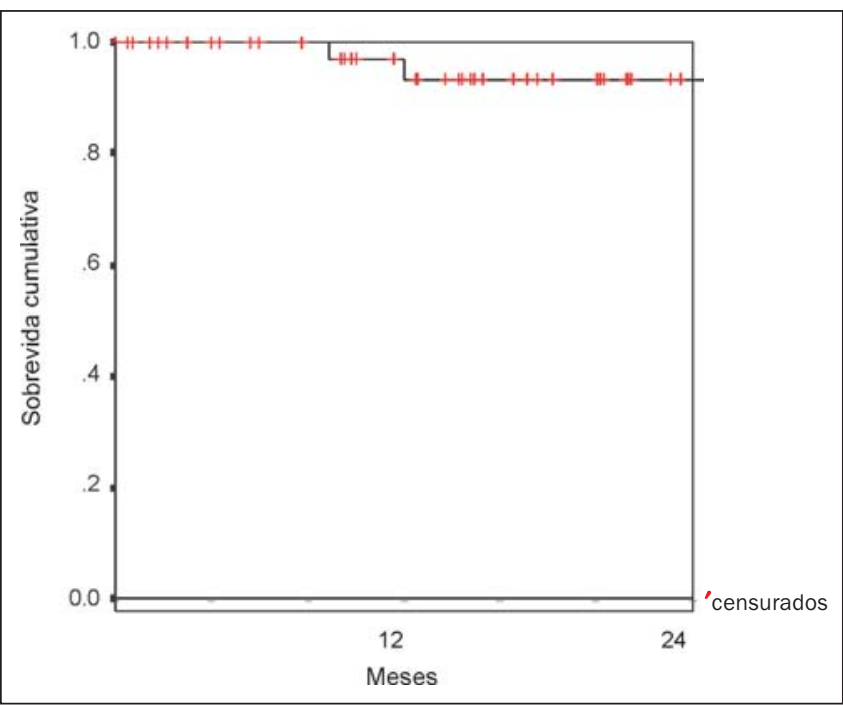

Figura 1. Sobrevida livre de doença atuarial em dois anos.

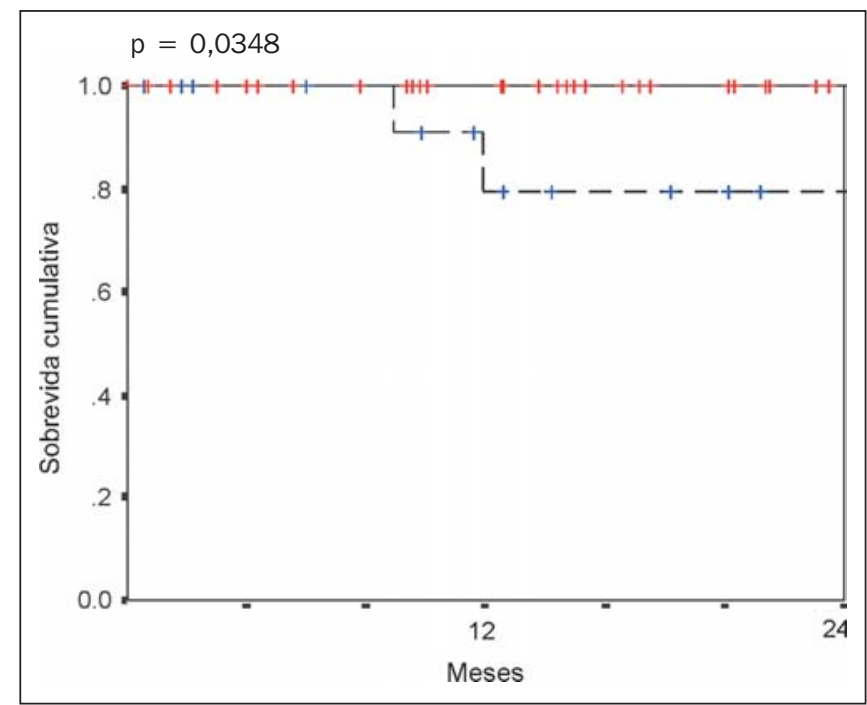

Figura 2. Sobrevida livre de doença atuarial em dois anos segundo a altura da lesão maior ou menor que $6 \mathrm{~mm}$.
Tabela 2 Análise univariada.

\begin{tabular}{|c|c|c|c|c|}
\hline \multirow{2}{*}{\multicolumn{2}{|c|}{ Variável }} & \multicolumn{2}{|c|}{ Falha local } & \multirow{2}{*}{$p^{*}$} \\
\hline & & $\mathrm{N}$ & $\%$ & \\
\hline Sexo & $\begin{array}{l}\text { Feminino } \\
\text { Masculino }\end{array}$ & $\begin{array}{l}1 / 31 \\
2 / 18\end{array}$ & $\begin{array}{r}3,2 \% \\
11,1 \%\end{array}$ & 0,8032 \\
\hline Idade (anos) & $\begin{array}{l}\leq 55 \\
>55\end{array}$ & $\begin{array}{l}1 / 21 \\
2 / 28\end{array}$ & $\begin{array}{l}4,7 \% \\
7,2 \%\end{array}$ & 0,7419 \\
\hline Tratamento (horas) & $\begin{array}{l}\leq 134 \\
>134\end{array}$ & $\begin{array}{l}0 / 18 \\
3 / 31\end{array}$ & $\overline{9,6 \%}$ & 0,3020 \\
\hline Dose na base (Gy) & $\begin{array}{l}\leq 340 \\
>340\end{array}$ & $\begin{array}{l}1 / 22 \\
2 / 27\end{array}$ & $\begin{array}{l}4,5 \% \\
7,4 \%\end{array}$ & 0,0951 \\
\hline Dose a 5 mm (Gy) & $\begin{array}{l}\leq 100 \\
>100\end{array}$ & $\begin{array}{l}1 / 28 \\
2 / 21\end{array}$ & $\begin{array}{l}3,5 \% \\
9,5 \%\end{array}$ & 0,0862 \\
\hline Dose no ápice (Gy) & $\begin{array}{l}\leq 100 \\
>100\end{array}$ & $\begin{array}{l}2 / 40 \\
1 / 9\end{array}$ & $\begin{array}{r}5,0 \% \\
11,1 \%\end{array}$ & 0,4604 \\
\hline Altura do tumor (mm) & $\begin{array}{l}\leq 6 \\
>6\end{array}$ & $\begin{array}{l}0 / 31 \\
3 / 18\end{array}$ & - & 0,0348 \\
\hline
\end{tabular}

*Teste exato de Fisher. Intervalo de confiança: 95\%.

anos sessenta, devido à perda da visão naquele lado e pelos quase $50 \%$ de taxa de mortalidade em cinco anos em conseqüência de metástases ${ }^{(4)}$.

A visão útil é preservada nos olhos em que os tumores surgem em uma localização favorável com respeito ao disco ótico ou mácula, porém, cerca de $16,3 \%$ destes pacientes necessitam, subsequientemente, de enucleação por causa de glaucoma ou progressão tumoral $^{(\mathbf{5})}$.

Como nenhum dos diferentes tratamentos oferece vantagem de sobrevida, um fa- tor importante na escolha da terapêutica a ser empregada é o impacto na qualidade de vida e na possibilidade de resgate terapêutico. Uma pesquisa recente reportou que a escolha da modalidade de tratamento para MC não pareceu estar associada a grandes diferenças na qualidade da vida, quando avaliada com seguimento em longo prazo, porém a possibilidade em adotar-se estratégias passíveis de resgate parece ser a mais bem aceita na atualidade ${ }^{(\mathbf{6})}$.

Pela falta de dados da literatura em relação aos prováveis caracteres clínicos implicados no prognóstico dos pacientes, utilizamos, em nossa análise, como níveis de corte, os valores medianos encontrados em relação a: idade, tempo de tratamento, altura e medida da base das lesões.

Para pacientes submetidos a tratamento conservador, a progressão da lesão depois de realizada braquiterapia deve ser registrada com fotografias consecutivas, ultrasom e transiluminação. A redução da lesão, após a terapêutica conservadora, qualquer que seja a empregada, depende de características individuais do tumor e do hospedeiro.

Cruess et al. avaliaram o comportamento das lesões de 100 pacientes portadores de MC tratados sucessivamente com braquiterapia, usando placas de cobalto-60, e verificaram que metade das lesões regrediu lentamente, persistindo como massa residual com aproximadamente $50 \%$ da espessura do tumor original após 54 meses $^{(7)}$.

Augsburger et al., em publicação recente, não observaram diferença na taxa de mortalidade doença-específica em 15 anos dos pacientes portadores de MC tratados por enucleação ou braquiterapia, após eliminação de variáveis clínicas individuais ${ }^{(8)}$.

Atualmente, o iodo-125 ou rutênio-106 são os isótopos preferenciais na realização da braquiterapia oftalmológica, em virtude da sua energia mais baixa, permitindo uma melhor proteção radiológica, tanto para a equipe envolvida no procedimento 
quanto para os tecidos normais intra e extra-oculares ${ }^{(9)}$.

Em conclusão, a nossa experiência inicial com braquiterapia oftalmológica utilizando o iodo-125 levou a uma alta taxa de controle local, com possibilidade de cirurgia de resgate para os pacientes que não alcançaram controle local. Observamos, ainda, ser a altura da lesão fator prognóstico relacionado ao controle local.

\section{REFERÊNCIAS}

1. Earle JD. Results from the Collaborative Ocular Melanoma Study (COMS) of enucleation versus preoperative radiation therapy in the management of large ocular melanomas. Int J Radiat Oncol Biol Phys 1999;43:1168-9.

2. Shields CL, Shields JA, Gunduz K, Freire JE, Mercado G. Radiation therapy for uveal malignant melanoma. Ophthalmic Surg Lasers 1998;29:397409.

3. Collaborative Ocular Melanoma Study Group Design and Methods of a Clinical Trial for a Rare Condition, The Collaborative Ocular Melanoma Study, COMS Report No. 3. Control Clin Trials 14 1993: 362-91.

4. Foss AJ, Lamping DL, Schroter S, Hungerford J. Development and validation of a patient based measure of outcome in ocular melanoma. $\mathrm{Br} \mathrm{J}$ Ophthalmol 2000;84:347-51.

5. Finger PT. Radiation therapy for choroidal melanoma. Surv Ophthalmol 1997;42:215-32.
6. Munzenrider JE. Uveal melanomas. Conservation treatment. Hematol Oncol Clin North Am 2001; 15 : 389-402.

7. Cruess AF, Augsburger JJ, Shields JA, Brady LW, Markoe AM, Day JL. Regression of posterior uveal melanomas following cobalt-60 plaque radiotherapy. Ophthalmology 1984;91:1716-9.

8. Augsburger JJ, Correa ZM, Freire J, Brady LW. Long-term survival in choroidal and ciliary body melanoma after enucleation versus plaque radiation therapy. Ophthalmology 1998;105:1670-8.

9. Petrovich Z, Astrahan M, Luxton G, Shields JA, Shields CL, Brady LW. Primary melanoma of the uvea: radioactive plaque therapy and other treatment modalities. In: Alberti WE, Sagerman RH, eds. Radiotherapy of intraocular and orbital tumors. New York: Springer-Verlag, 1993:33-44. 